\title{
A Mathematical Model of Skeletal Muscle and Numerical Simulations of Its Response under Stretching*
}

\author{
Hiroshi YAMADA** and Janusz KAJZER***
}

\begin{abstract}
A mathematical model is formulated for activated muscles under stretching. "Yielding", which is characterized as the decrease in the rate of muscle force during stretching, is reported in the literature for activated cat soleus muscles. We hypothesize that such "yielding" mainly results from the change of an active state that is determined by step functions of activation stimulus which reflects the properties of muscle conditions. The activation stimulus increases when the muscle begins to be stretched, and it decreases after a short duration at the start of stretching and when the stretching is stopped. The effect of velocity and stimulation rate is expressed by determining the change of activation stimulus systematically. The simulation results show that the "yielding" is described by a simple frame of the model. The model might be applied to simulate the behavior of activated muscles during stretching such as walking and sports.
\end{abstract}

Key Words: Biomechanics, Constitutive Equation, Numerical Analysis, Skeletal Muscle, Neural Stimulation, Activation Stimulus, Stretching

\section{Introduction}

The muscles in various parts of the human body are often stretched during the contracted state in daily lives, sports activities and accidents. While experimental and theoretical studies of contracted muscles have mainly focused on the behavior of shortening, there are some reports of muscles during lengthening dealing with the force-velocity characteristics ${ }^{(1),(2)}$. However, this curve of force-velocity characteristics does not always represent the deformation behavior of lengthening.

Decrease of force increment rate has been reported in the activated cat soleus muscles during lengthening with a constant velocity ${ }^{(1),(3)}$. This phenomenon is called "yielding" because of the similarity of the shape of force-length curve of the muscle with a yielding

* Received 2nd March, 1999

** Department of Micro System Engineering, Graduate School of Engineering, Nagoya University, Furo-cho, Chikusa-ku, Nagoya 464-8603, Japan. E-mail: yamada@mech.nagoya-u.ac.jp

*** Department of Mechanical Engineering, Graduate School of Engineering, Nagoya University part of the stress-strain diagram of metals, but there is no similarity in material properties. The "yielding" is observed in the muscle which is originally under steady state for a sufficient period of time and not in newly recruited muscle fibers ${ }^{(4)}$. Winters proposed a mathematical model to describe the "yielding" phenomenon $^{(4)}$. This model requires an active state level change beforehand to express the variable of yielding, which restricts its application ${ }^{(5)}$.

Winters and Stark proposed an evolution equation of an internal variable of active muscle state to describe the dynamics of activation ${ }^{(6),(7)}$. This equation can relate the active state variable with an idealized neurocontroller input signal and makes it possible to include the effect of signals from the muscle receptors.

In the current study we hypothesized that the "yielding" is a result of the change of an active state level during muscle stretching. Based on this idea we introduced a series of step changes of activation stimulus to determine the active state of the muscle model. Then we carried out numerical simulations of the "yielding" phenomenon to validate this concept. 


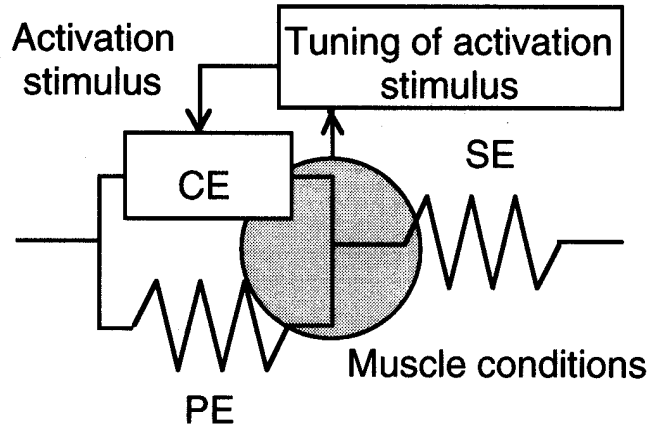

Fig. 1 Muscle model

\section{A Mathematical Model of Muscles}

The present model is a combination of a Hillbased model ${ }^{(8)}$ and an activation stimulus model which determines the active state level of the muscle as illustrated in Fig. 1.

The model consists of three elements, i.e., parallel elastic element (PE), series elastic element (SE) and contractile element $(\mathrm{CE})$. The total length is the sum of the length of $\mathrm{CE}\left(L^{C E}\right)$ and that of $\mathrm{SE}\left(L^{S E}\right)$ as Eq. (1). To remove the arbitrary division of lengths between $\mathrm{CE}$ and $\mathrm{SE}$, we assume the ratio $\left(\rho_{0}\right)$ of the length of $\mathrm{CE}$ to the whole muscle length at the stress ${ }^{-}$ free state as Eq. (2).

$$
\begin{aligned}
& L^{\text {total }}=L^{C E}+L^{S E} \\
& \rho_{0}=L_{0}^{C E} / L_{0}^{\text {total }}
\end{aligned}
$$

The muscle force $\left(F_{m u s}\right)$ is the sum of the force of $\mathrm{CE}\left(F_{C E}\right)$ and that of $\mathrm{PE}\left(F_{P E}\right)$ as Eq. $(3)$, and it is equal to the force of SE $\left(F_{S E}\right)$.

$$
F_{\text {mus }}=F_{C E}\left(N_{a}, \varepsilon, \dot{\varepsilon}\right)+F_{P E}(\varepsilon)=F_{S E}
$$

The force of $\mathrm{CE}$ is assumed to be the product of the active state $\left(N_{a}\right)$, the maximal isometric force $\left(F_{l}\right)$ and the effect of strain rate $\left(F_{v}\right)$ as in Eq. $(4)^{(4)}$.

$F_{C E}\left(N_{a}, \varepsilon, \dot{\varepsilon}\right)=N_{a}(u[\tau ; \tau \leq t]) \cdot F_{l}(\varepsilon) \cdot F_{v}(\dot{\varepsilon}) \quad$ (4)

The active state is determined by the activation stimulus $(u)$, and is expressed with a set of differential equations ${ }^{(6),(7)}$ as

$$
\left\{\begin{array}{l}
T_{n e} \cdot \dot{N}_{e}(t)=u(t)-N_{e}(t) \\
T_{a} \cdot \dot{N}_{a}(t)=N_{e}(t)-N_{a}(t)
\end{array}\right.
$$

where the varaible $N_{e}$ can be eliminated to obtain a second order differential equation of $N_{a} . T_{n e}$ and $T_{a}$ are time constants.

To describe the force change during stretching, the activation stimulus is modeled as a series of step functions which depend on muscle conditions. The activation stimulus depends on the muscle conditions as well as the neural stimulation. In the case the afferent nerve is intact it reflects the signals from the receptors in the muscle and this neural feedback may work to protect the muscle from an excessive elongation or damage. These receptors, i.e., the neuromuscular spindles, have the characteristics of phasic receptor responding to the change of external stimulation and tonic receptor responding to the steady stimulation level ${ }^{(9)}$. During muscle stretching, the spindles generate impulses frequently in the initial phase and then decreases in frequency. It is also known that the larger stretching velocity causes a higher frequency of impulses in the initial phase.

The maximal isometric force $\left(F_{l}\right)$ in Eq. $(4)$ is a function of the strain of $\mathrm{CE}$ and not of the total strain of the muscle $\left(\varepsilon^{\text {total }}\right)$. Thus, we can express an apparent relationship $\left(F_{l}^{a p}\right)$ as a function of $\varepsilon^{\text {total }}$ by

$$
F_{l}^{a p}\left(\varepsilon^{\text {total }}\right)=F_{\max }-C_{l}\left(\varepsilon^{\text {total }}-\varepsilon_{\max }^{\text {total }}\right)^{2}
$$

where $F_{\max }$ and $\varepsilon_{\max }^{\text {total }}$ are the peak force of the maximal isometric force and the corresponding strain of the muscle, respectively, and $C_{l}$ is a material constant. The relationship between $F_{\max }$ and the strain of $\mathrm{CE}$ can be derived from the balance of force between $\mathrm{CE}$ and SE under arbitrary lengths.

The effect of strain rate on the force of $\mathrm{CE}^{(6),(10)}$, which is normalized to be one at zero strain-rate, is described by

$$
\begin{aligned}
& F_{v}=\left\{\begin{array}{r}
\left(1+\dot{\varepsilon}^{*}\right) /\left(1-\dot{\varepsilon}^{*} / C_{\text {short }}\right), \\
-1 \leq \dot{\varepsilon}^{*} \leq 0 \\
\left(1+\dot{\varepsilon}^{*} C_{\text {mvl }} / C_{\text {leng }}\right) /\left(1+\dot{\varepsilon}^{*} / C_{\text {leng }}\right), \\
0<\dot{\varepsilon}^{*}
\end{array}\right. \\
& \dot{\varepsilon}^{*}=v /\left|v_{\text {max }}\right|=\dot{\varepsilon} /\left|\dot{\varepsilon}_{\max }\right|
\end{aligned}
$$

where $F_{v}$ reaches the level of $C_{m v l}$ when the strain rate is infinitively large. $C_{\text {short }}$ and $C_{\text {leng }}$ are material constants, and $v_{\max }$ the maximal shortening velocity. The experimental results of force-velocity relationship reported in the literature ${ }^{(1)}$ are not for the contractile element but for the whole muscle. In the current model, however, we assume that the force of $\mathrm{CE}$ follows the relationship ${ }^{(8)}$ as a function of the strain rate of $\mathrm{CE}$.

The force-strain relationship of PE is generally expressed by an exponential function when the strain is positive. The force is zero when the strain is negative. In the present study we will not show the equation explicitly because the strain range in the numerical simulations is always negative.

The force-strain relationship of $\mathrm{SE}^{(6)}$ is expressed by

$$
F_{S E}=\left\{\begin{array}{c}
\frac{F_{\max }}{\exp \left(C_{S E}\right)-1}\left[\exp \left(\frac{\varepsilon \cdot C_{S E}}{S E_{\max }}\right)-1\right], 0 \leq \varepsilon \leq \varepsilon_{\text {elast }} \\
k_{S E} \cdot\left(\varepsilon-\varepsilon_{\text {elast }}\right)+F_{S E}\left(\varepsilon_{\text {elast }}\right), \quad \varepsilon>\varepsilon_{\text {elast }}
\end{array}\right.
$$

when the strain is positive where

$$
k_{S E}=\frac{C_{S E}}{S E_{\max }} \frac{F_{\max }}{\exp \left(C_{S E}\right)-1} \exp \left(\frac{\varepsilon_{\text {elast }} \cdot C_{S E}}{S E_{\max }}\right)
$$

and $C_{S E}, S E_{\max }$ and $\varepsilon_{\text {elast }}$ are material constants. The force of $\mathrm{SE}$ is zero when the strain is negative. 
Table 1 Experimental conditions ${ }^{(3)}$ and activation stimuli

\begin{tabular}{|c|c|c|c|c|c|c|}
\hline & Freq. & Vel. & \multicolumn{4}{|c|}{ Level (Start time (s)) of activation stimuli } \\
\cline { 2 - 7 } & $\mathrm{Hz}$ & $\mathrm{mm} / \mathrm{s}$ & Step 1 & Step 2 & Step 3 & Step 4 \\
\hline 1 & 8 & 5 & $0.74(\mathrm{t}<0)$ & $0.77(0.0)$ & $0.69(0.09)$ & $0.61(0.2)$ \\
\hline 2 & 8 & 10 & $0.74(\mathrm{t}<0)$ & $0.83(0.0)$ & $0.68(0.05)$ & $0.59(0.2)$ \\
\hline 3 & 8 & 30 & $0.74(\mathrm{t}<0)$ & $0.95(0.0)$ & $0.63(0.03)$ & $0.53(0.2)$ \\
\hline 4 & 8 & 12.5 & $0.74(\mathrm{t}<0)$ & $0.83(0.0)$ & $0.68(0.05)$ & $0.59(0.16)$ \\
\hline 5 & 10 & 12.5 & $0.80(\mathrm{t}<0)$ & $0.96(0.0)$ & $0.77(0.05)$ & $0.60(0.16)$ \\
\hline
\end{tabular}

\section{Numerical Simulations}

\section{1 Methods}

The proposed model of yielding was validated by comparing the numerical simulation results with experimental ones from the literature. We chose the experimental results of force changes in cat soleus muscles under various constant stimulation rates and constant stretching velocities as shown in the left three columns in Table $1^{(3)}$. The subdivided ventral roots were stimulated electrically to develop the force in the muscle where the afferent nerve was not intact. The muscle was initially shortened by $12 \mathrm{~mm}$ under a constant rate of electrical stimulation through the nerves and was stretched with a constant velocity for 0.2 second (\# 1-3 in Table 1) and 0.16 second (\# 4, 5 in Table 1) by maintaining the rate of stimulation. After the stretch, the muscle length was kept constant. The force levels of one set of experiments $(\# 1-3)$ and another set of experiments $(\# 4,5)$ showed a large discrepancy. Then we transformed the scale of the latter force levels as shown in Fig. 4 (b) to make the force levels close enough between \# 2 and \# 4 whose conditions are similar. To make the characteristics of the experimental results clear, we also matched the force levels of \#1-3 at the start point of stretching whose original force data are slightly different.

The parameters in the model were determined as $L_{0}{ }^{\text {total }}=60(\mathrm{~mm}), \rho_{0}=0.9, T_{n e}=0.025(\mathrm{~s})$,

$$
T_{a}=0.015(\mathrm{~s}), \varepsilon_{\text {max }}^{\text {total }}=0.053 \text {, }
$$$$
F_{\text {max }}=20.6(\mathrm{~N}), C_{l}=120(\mathrm{~N}), v_{\max }=-100(\mathrm{~mm} / \mathrm{s}) \text {, }
$$$$
C_{\text {short }}=0.15, C_{\text {leng }}=0.03 \text {, }
$$$$
C_{m v l}=1.17, C_{S E}=7.0, S E_{\max }=0.05, \varepsilon_{\text {elast }}=0.02
$$

In the above parameters, the value of $L_{0}{ }^{\text {total }}$ was assumed due to the lack of information of the length in the above experiment. $\rho_{0}$ was assumed to eliminate any arbitrariness as mentioned above. The excitation time constant $T_{n e}$ and the activation time constant $T_{a}$ were determined based on the literature ${ }^{(6),(7)}$. Figure 2 (a) shows the force-length relationships of the whole muscle (solid line) and SE (dashed line). A circle denotes the maximal isometric force of cat soleus muscles during $35 \mathrm{~Hz}$ stimulation ${ }^{(11)}$. The values of $F_{\max }, C_{l}$ and $\varepsilon_{\text {max }}^{\text {total }}$ in Eq. $(6)$ were determined to fit this result. Figure 2(b) shows the force-velocity
Table 2 Characteristics of activation stimuli

\begin{tabular}{|c|c|c|c|c|c|}
\hline & $\begin{array}{c}\text { Level of } \\
\text { Step 1 }\end{array}$ & $\begin{array}{c}\text { Level of } \\
\text { Step 2 }\end{array}$ & $\begin{array}{c}\text { Duration of } \\
\text { Step 2 }\end{array}$ & $\begin{array}{c}\text { Level of } \\
\text { Step 3 }\end{array}$ & $\begin{array}{c}\text { Level of } \\
\text { Step 4 }\end{array}$ \\
\hline Larger vel. & - & increase & decrease & decrease & decrease \\
\hline Larger freq. & increase & increase & no change & increase & increase \\
\hline
\end{tabular}

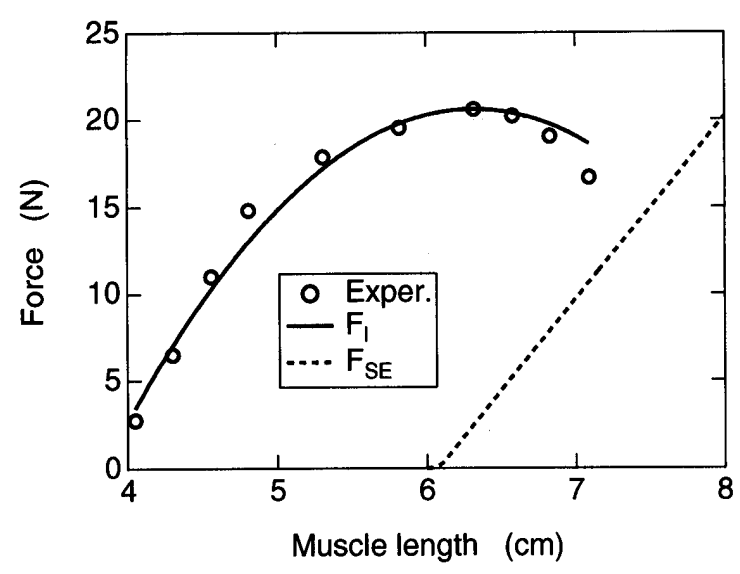

(a) Force-length relationships

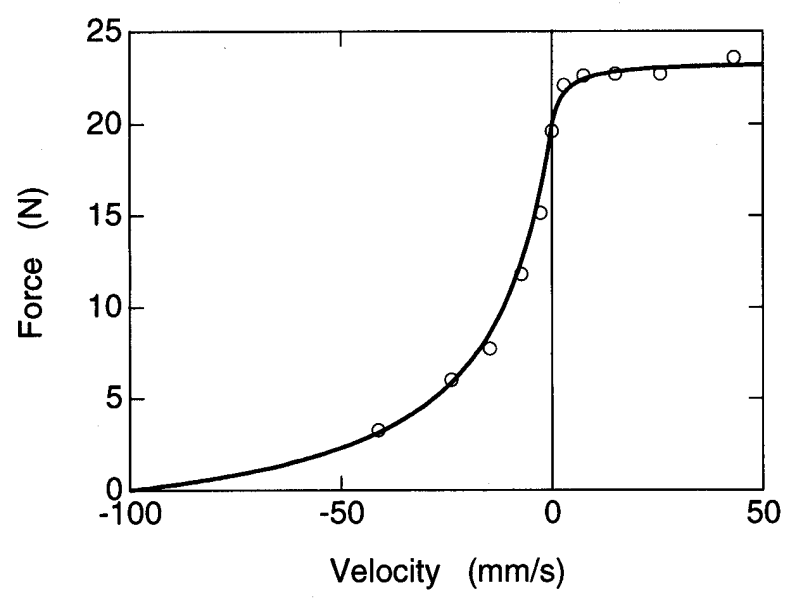

(b) Force-velocity relationship

Fig. 2 Force-length and force-velocity relationships ${ }^{(1),(11)}$

relationship of $\mathrm{CE}$ (solid line). A circle denotes the force at each velocity of cat soleus muscles during 35 $\mathrm{Hz}$ stimulation ${ }^{(1)}$. The values of $v_{\max }, C_{\text {short }}, C_{\text {leng }}$ and $C_{m v l}$ in Eqs. ( 7 ) and ( 8 ) were determined to match these characteristics. $C_{S E}, S E_{\max }$ and $\varepsilon_{\text {elast }}$ were also determined based on the literature ${ }^{(6),(7)}$.

The activation stimuli were determined as indicated in Table 1 for the stimulation rate and stretching velocity to describe the deformation behavior of the muscles in Fig. 3(b) and Fig. 4(b). The level of the activation stimulus was determined first to match the force level before stretching at a constant electrical stimulation rate through the nerves. Then the level and the duration of the second step of the stimulus were determined to describe the increasing part of the force change under stretching. The larger velocity increases the stimulus level and decreases the duration. The larger stimulation rate increases the 


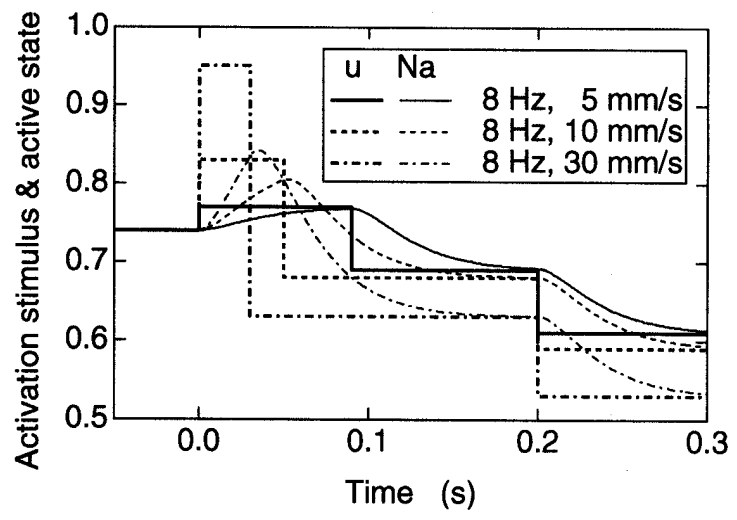

(a) Activation stimulus and active state

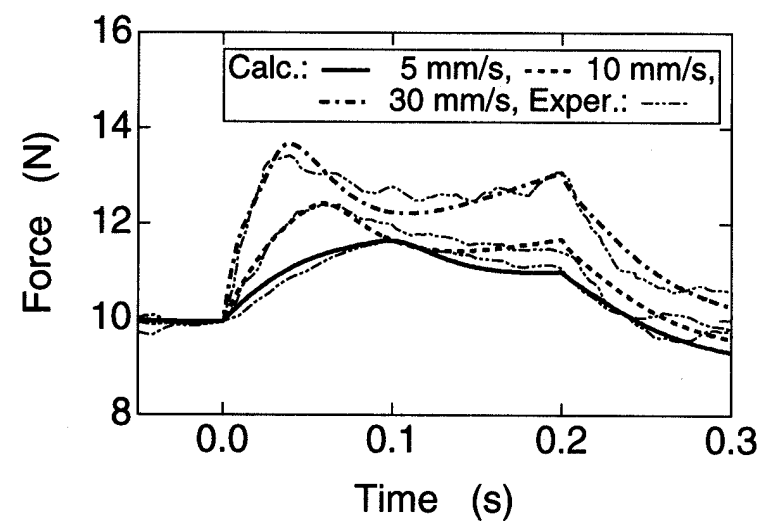

(b) Force change

Fig. 3 Simulation results of changes in activation stimulus, active state and force of muscles under conditions \# $1-3$ in Table $1^{(3)}$

stimulus level in all the steps while keeping the duration constant. The decrease of the level of activation stimulus occurs twice, the first at peak force during stretching and second after finishing the stretching. The characteristics of the activation stimulus are summarized as Table 2.

\section{2 Results and discussions}

Figure 3(a) shows the step changes of the activation stimulus and the corresponding changes in the active state. Figure $3(\mathrm{~b})$ gives the comparisons of the force change of the cat soleus muscles between the numerical simulations and experiments in the literature ${ }^{(3)}$. The conditions are $8 \mathrm{~Hz}$ electrical stimulation through the nerves and 0.2 -second stretching with a constant velocity of 5,10 or $30 \mathrm{~mm} / \mathrm{s}^{(3)}$. Figure 4 shows the results of the same variables under $8 \mathrm{~Hz}$ or $10 \mathrm{~Hz}$ electrical stimulation through the nerves and a 0.16 -second stretching with a constant velocity of $12.5 \mathrm{~mm} / \mathrm{s}$. The results of the active state show that the peak of the active state at the initial stage of stretching does not coincide with the end of the activation stimulus step. It tends to delay when the duration of the activation stimulus step becomes smaller. The results of force changes show that the proposed model

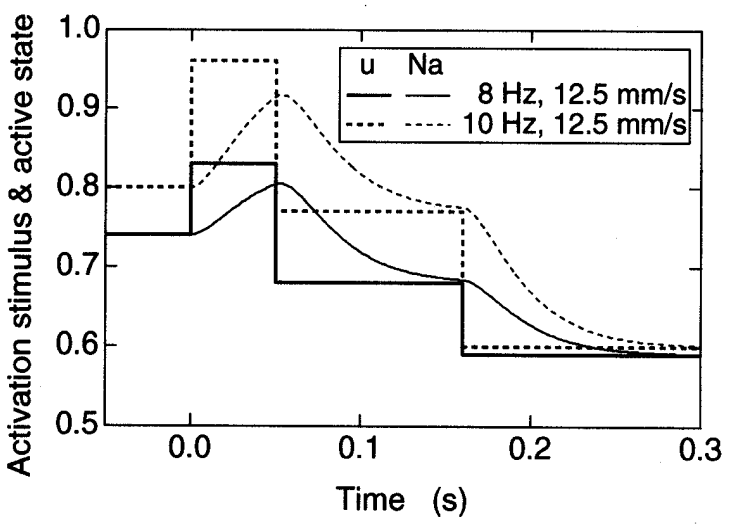

(a) Activation stimulus and active state

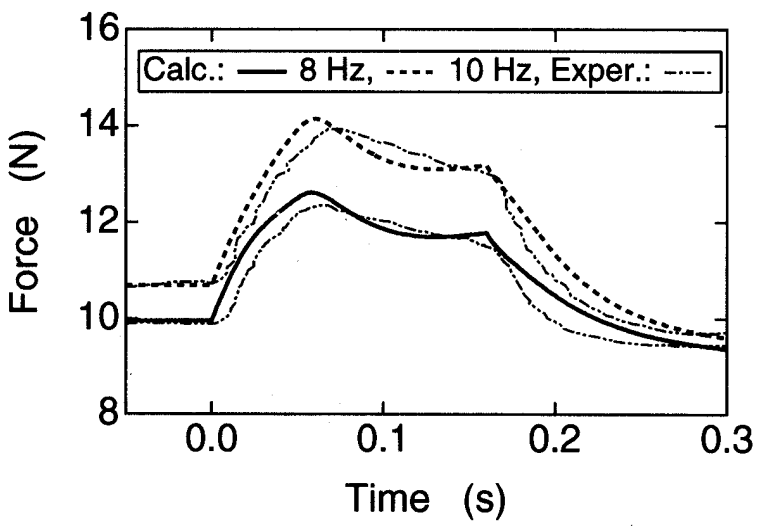

(b) Force change

Fig. 4 Simulation results of changes in activation stimulus, active state and force of muscles under conditions \# 4, 5 in Table $1^{(3)}$

well describes the effect of stretching velocities and stimulation rate on force changes, indicating the muscle yielding by changing the activation stimulus step systematically as characterized in Table 2. The tendency of the activation stimulus in these simulations is consistent with the observations of the neuromuscular system in the literature ${ }^{(9)}$. Any muscle response in the range of stretching velocity and stimulation rate can be interpolated with a series of step functions of the activation stimulus. The model also describes the decrease and re-increase of force under the larger stretching velocity of $30 \mathrm{~mm} / \mathrm{s}$ with a stimulation rate of $8 \mathrm{~Hz}$ while the force in the experiment tends to decrease under smaller stretching velocities. The reason why muscle force increases during the period of the decrease of active state is that the increase in the maximal isometric force is larger compared with the decrease in the active state level. The increase rate of the maximal isometric force is larger for a higher muscle velocity.

Winters' equation for the variable of yielding consists of various parameters, e.g., muscle velocity, time derivative of active state and its maximum ${ }^{(4)}$. In his model, the determination of active state and its 


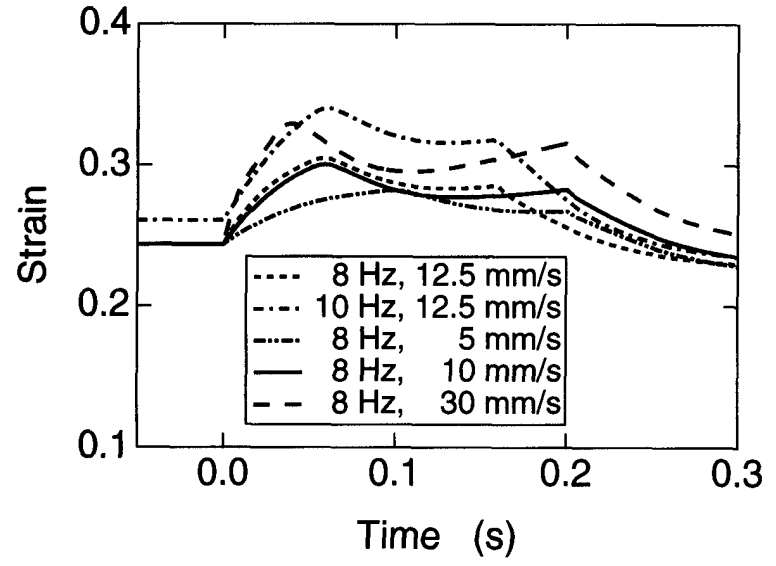

(a) Series elastic element

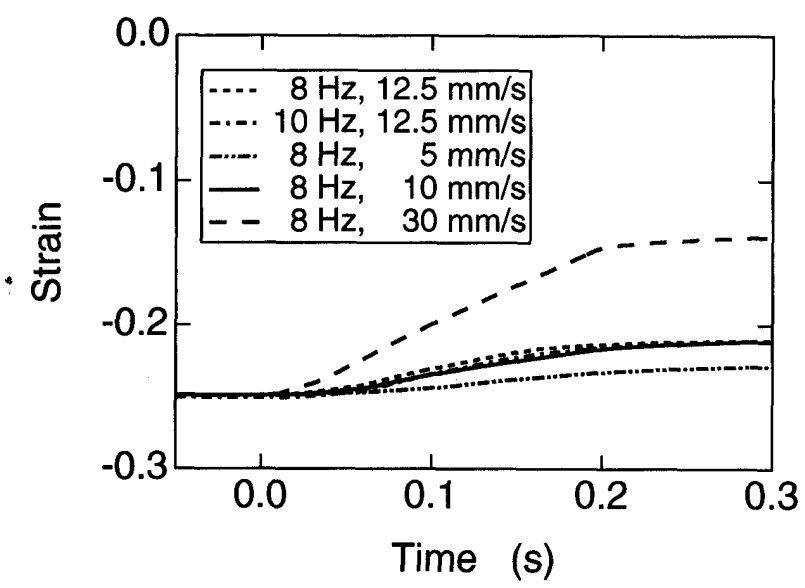

(b) Contractile element

Fig. 5 Simulation results of strain changes of the series elastic element and the contractile element

time derivative is the key, and this is more difficult than the determination of the activation stimulus steps in our model. Though our model is not fully validated for all possible loading conditions (variable stretching velocity), the activation stimulus is the only parameter necessary to be determined under each loading condition.

Figure 5(a) and Fig. 5(b) show the strain changes of $\mathrm{SE}$ and $\mathrm{CE}$ for \#1-5, respectively. Because the strain of SE corresponds to the force of $\mathrm{SE}$, the increase in $\mathrm{SE}$ strain means the increase in muscle force. A decrease in SE strain does not directly decrease the length of the whole muscle because the length of the SE part is assumed to be small enough compared with the $\mathrm{CE}$ part. The $\mathrm{CE}$ strain, on the other hand, always increases during muscle stretching even when the muscle force decreases.

Figure 6 shows the force-displacement relationship of the muscle by converting the axis of time in Fig. 3(b) to the axis of muscle displacement. The experimental results show that the forces under three different velocities are almost the same during force

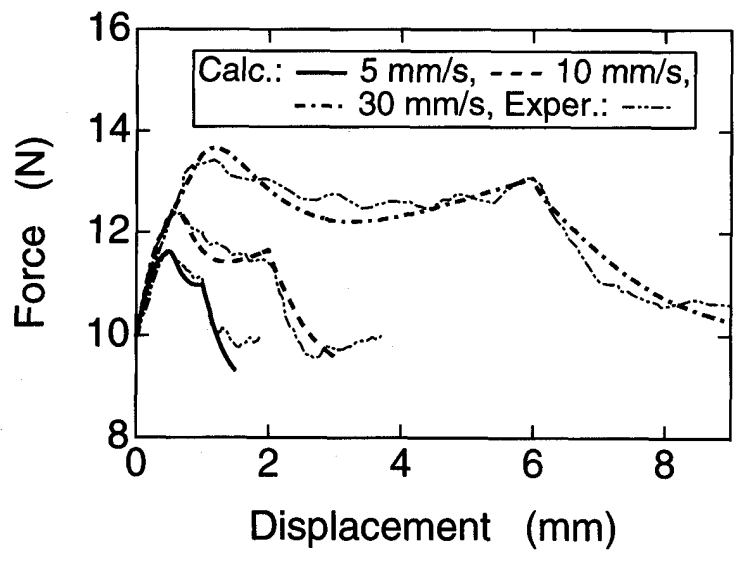

Fig. 6 Force-displacement relationship

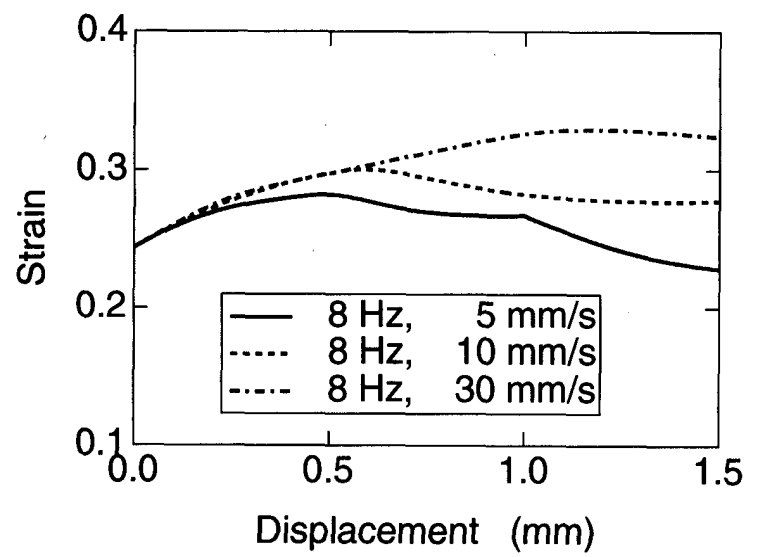

Fig. 7 Relationship between strains of the series elastic element and muscle displacement

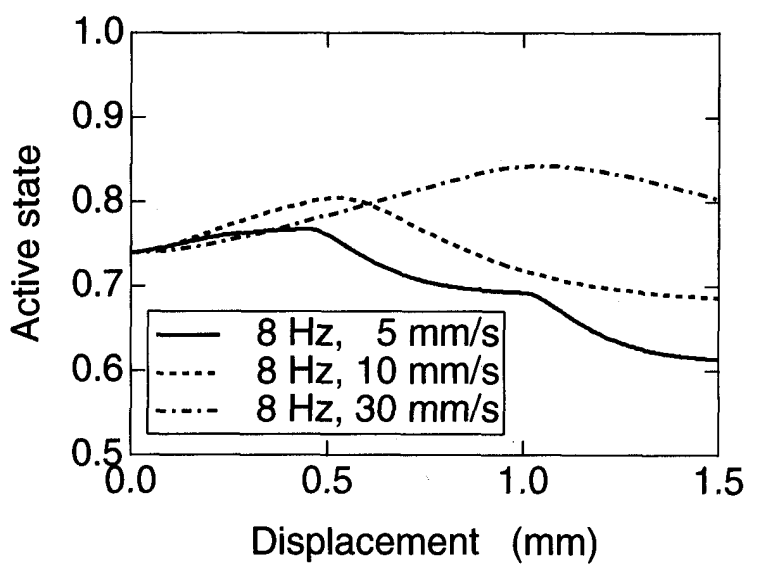

Fig. 8 Relationship between the active state level of the contractile element and muscle displacement

development. The model describes this relationship, too. Figure 7 gives the SE strain for muscle displacement in the same condition as in Fig. 6, which makes the similarity of force changes clear. The results show the coincidence of the curves between the conditions of the velocities of 10 and $30 \mathrm{~mm} / \mathrm{s}$ and slightly smaller strain under the velocity of $5 \mathrm{~mm} / \mathrm{s}$. Figure 8 shows the relationship between the active state level 
and the muscle displacement under the same conditions indicated in Fig. 6. Compared with Fig. 3(a), which shows the different time-history of active state levels, the displacement-active state functions are quite similar during force increases in the muscle. These results indicate that the determination of the activation stimulus should be carried out to match the force-displacement relationship of the muscle whose initial part is a single curve under various velocities.

\section{Conclusions}

We formulated a mathematical model of activated muscles under stretching by hypothesizing that the "yielding" resulted from the change in an active state level. The change of the active state was determined by a series of step functions of activation stimulus which reflects the muscle conditions in the test setup ${ }^{(3)}$. The simulation results showed that the "yielding" phenomenon could be described by a simple frame of the model. The effects of stretching velocity and stimulation rate were also expressed by systematically determining the change of activation stimulus. The model might be applied to simulate the behavior of activated muscles during stretching such as in walking and sports activities.

\section{References}

(1) Joice, G.C., Rack, P.M.H. and Westbury, D.R., The Mechanical Properties of Cat Soleus Muscle during Controlled Lengthening and Shortening Movements, J. Physiol., Vol. 204 (1969), p. 461474.

(2) Winter, D.A., Biomechanics and Motor Control of Human Movement, (2nd ed.), (1990), p. 165-189,
John Wiley and Sons.

(3) Cordo, P.J. and Rymer, W.Z., Contributions of Motor-Unit Recruitment and Rate Modulation to Compensation for Muscle Yielding, J. Neurophysiol., Vol. 47 (1982), p. 799-809.

(4) Winters, J.M., Hill-Based Muscle Models: A Systems Engineering Perspective, in Multiple Muscle Systems: Biomechanics and Movement Organization, Winters, L.M. and Woo, S.L-Y., ed., (1990), p. 69-93, Springer-Verlag.

(5) Yamada, H., Takeuchi, H., Nishio, K., Wittek, A. and Kajzer, J., Muscle Yielding PhenomenonModeling and Simulations of the Response under Transient Stretching, Prepr. JSME, No. 983-1 (1998), p. 33-34.

(6) Winters, J.M. and Stark, L., Estimated Mechanical Properties of Synergistic Muscles Involved in Movements of a Variety of Human Joints, J. Biomech., Vol. 21, No. 12 (1988), p. 1027-1041.

(7) Wittek, A. and Kajzer, J., Modelling of Muscle Influence on the Kinematics of the Head-Neck Complex in Impacts, Memoirs of the School of Eng., Nagoya University, Vol. 49, No. 2 (1997), p. 151-205.

(8) Hill, A.V., The Heat of Shortening and the Dynamic Constants of Muscle, Proc. R. Soc. Lond., Ser. B., Vol. 126 (1938), p. 136-195.

(9) Mashima, H., Physiology, (in Japanese), (1986), p. 97-217, Bunkodo.

(10) Winters, J.M. and Stark, L., Analysis of Fundamental Human Movement Patterns Through the Use of In-Depth Anatagonic Muscle Models, IEEE, Trans. Biomed. Eng., Vol. 32, No. 10 (1985), p. 826-839.

(11) Rack, P.M.H. and Westbury, D.R., The Effects of Length and Stimulus Rate on Tension in the Isometric Cat Soleus Muscle, J. Physiol., Vol. 204 (1969), p. 443-460. 